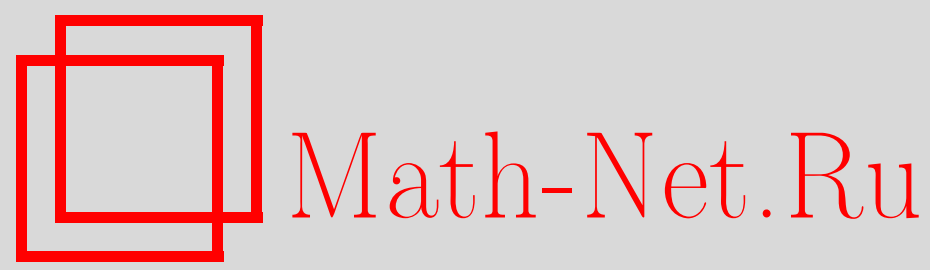

П. Л. Гуревич, Обобщенные решения нелокальных эллиптических задач, Матем. заметки, 2005, том 77, выпуск 5, 665-682

DOI: https://doi.org/10.4213/mzm2525

Использование Общероссийского математического портала Math-Net.Ru подразумевает, что вы прочитали и согласны с пользовательским соглашением http://www . mathnet.ru/rus/agreement

Параметры загрузки:

IP : 18.234 .197 .8

26 апреля 2023 г., 14:31:12

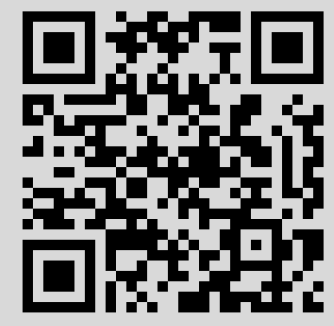




\section{ОБОБЩЕННЫЕ РЕШЕНИЯ НЕЛОКАЛЬНЫХ ЭЛЛИПТИЧЕСКИХ ЗАДАЧ}

\section{П. Л. Гуревич}

Рассмотрены эллиптические уравнения порядка $2 m$ с общими нелокальными краевыми условиями в плоской ограниченной области $G$ с кусочно-гладкой границей. Изучены обобщенные решения, принадлежащие пространству Соболева $W_{2}^{m}(G)$. Доказано, что неограниченный оператор, действующий в пространстве $L_{2}(G)$, соответствующий эллиптическому уравнению и определенный на функциях из пространства $W_{2}^{m}(G)$, удовлетворяющих однородньг нелокальным условиям, фредгольмов.

Библиография: 29 названий.

Введение. В одномерном случае нелокальные задачи изучали еще А. Зоммерфельд [1], Я. Д. Тамаркин [2], М. Пиконе [3]. В работе Т. Карлемана [4] ищется гармоническая в двумерной ограниченной области функция, на которую налагается нелокальное условие, связьвающее ее значения в различных точках границы. Другую постановку нелокальной задачи, возникающую в теории плазмы, рассмотрели А. В. Бицадзе и А. А. Самарский [5]: найти гармоническую в прямоугольнике функцию, удовлетворяющую нелокальным условиям на сдвигах границы; при этом сдвиги могут отображать точки гранищы внутрь области. Различные обобщения указанных задач рассматривали многие авторы [6]-[12].

Наиболее сложной оказьвается ситуация, когда носитель нелокальных членов имеет непустое пересечение с границей области: решения нелокальной задачи могут иметь степенные особенности вблизи некоторых точек даже при бесконечно гладкой гранище области и бесконечно дифференцируемой правой части [13], [14]. Поэтому для изучения таких задач применяют специальные весовые пространства, введенные В.А. Кондратьевьм при исследовании краевых задач в областях с особенностями на границе [15]. Наиболее полная теория в этом случае построена в работах А. Л. Скубачевского [13], [16]-[19].

Отметим, что интерес к нелокальным задачам в настоящее время вызван не только значительными теоретическими достижениями, но также важными приложениями, возникающими в биофизике, теории диффузионных процессов [20], теории плазмы [21] и т.д.

Работа выполнена при финансовой поддержке Российского фонда фундаментальных исследований, грант № 02-01-00312, Министерства образования РФ, грант № E02-1.0-131, и фонда INTAS, грант № YSF 2002-008. 
В работе изучаются обобщенные решения эллиптического уравнения порядка $2 m$ в двумерной области $G$, удовлетворяющие нелокальньм краевым условиям, которые заданы на частях $\Gamma_{j}$ границы области $\partial G=\cup \overline{\Gamma_{j}}$. Под обобщенными решениями понимаются функции из пространства Соболева $W^{m}(G)=W_{2}^{m}(G)$. Доказано, что неограниченньй оператор, действующий в пространстве $L_{2}(G)$ и соответствующий нелокальной эллиптической задаче, фредгольмов.

Заметим, что решение нелокальной задачи можно искать в пространстве "гладких" функций, а именно в пространстве Соболева $W^{2 m}(G)$ (см. [22], [23]) или в весовых пространствах $H_{a}^{2 m}(G)$, где

$$
\|u\|_{H_{a}^{k}(G)}=\left(\sum_{|\alpha| \leqslant k} \int_{G} \rho^{2(a-k+|\alpha|)}\left|D^{\alpha} u\right|^{2}\right)^{1 / 2}
$$

$k \geqslant 0$ целое, $a \in \mathbb{R}, \rho=\rho(y)=\operatorname{dist}(y, \mathscr{K}), \mathscr{K}=\cup \overline{\Gamma_{j}} \backslash \Gamma_{j}-$ конечное множество точек сопряжения нелокальных условий (см. [13], [17]). В обоих случаях задаче будет соответствовать ограниченньй оператор, фредгольмовость которого определяется спектральньми свойствами некоторых вспомогательных нелокальных задач с параметром. В свою очередь, на спектральные свойства вспомогательных задач влияют такие факторы, как значения коэффициентов в нелокальных условиях и геометрическая структура носителя нелокальных членов и границы области вблизи множества $\mathscr{K}$. Если же мы ищем обобщенные решения (т.е. функции из $W^{m}(G)$ ), то соответствующий неограниченньй оператор оказывается фредгольмовым вне зависимости от перечисленных вьше факторов.

Ранее вопрос о фредгольмовости неограниченного оператора в $L_{2}(G)$ изучался лишь в том случае, когда нелокальные условия заданы на сдвигах границы [19], или же в случае нелокального возмущения задачи Дирихле для уравнения второго порядка [11], [12]. Случай эллиптического уравнения порядка $2 m$ с общими нелокальными условиями рассматривается впервые.

\section{1. Постановка нелокальных задач в ограниченной области.}

1.1. Постановка задачи. Пусть $G \subset \mathbb{R}^{2}$ - ограниченная область с границей $\partial G$. Введем множество $\mathscr{K} \subset \partial G$, состоящее из конечного числа точек. Пусть $\partial G \backslash \mathscr{K}=\bigcup_{i=1}^{N} \Gamma_{i}$, где $\Gamma_{i}$ - открытые (в топологии $\partial G$ ) кривые класса $C^{\infty}$. Будем предполагать, что в окрестности каждой точки $g \in \mathscr{K}$ область $G$ совпадает с некоторьм плоским углом.

Обозначим через $\mathbf{P}\left(y, D_{y}\right)=\mathbf{P}\left(y, D_{y_{1}}, D_{y_{2}}\right)$ и $B_{i \mu s}\left(y, D_{y}\right)=B_{i \mu s}\left(y, D_{y_{1}}, D_{y_{2}}\right)$ дифференциальные операторы порядков $2 m$ и $m_{i \mu}\left(m_{i \mu} \leqslant m-1\right)$ соответственно с комплекснозначными коэффициентами класса $C^{\infty}$, а через $\mathbf{P}^{0}\left(y, D_{y}\right)$ и $B_{i \mu s}^{0}\left(y, D_{y}\right)$ их главные однородные части, $i=1, \ldots, N, \mu=1, \ldots, m, s=0, \ldots, S_{i}$. Здесь $D_{y}=\left(D_{y_{1}}, D_{y_{2}}\right)$, $D_{y_{j}}=-i \partial / \partial y_{j}$.

Сформулируем условия на операторы $\mathbf{P}\left(y, D_{y}\right)$ и $B_{i \mu 0}\left(y, D_{y}\right)$, которые будут соответствовать “локальной” эллиптической задаче. Предположим, что оператор $\mathbf{P}\left(y, D_{y}\right)$ собственно әллиптичен на $\bar{G}$; в частности, для всех $\theta \in \mathbb{R}^{2}$ и $y \in \bar{G}$ вьполнена оценка

$$
A^{-1}|\theta|^{2 m} \leqslant\left|\mathbf{P}^{0}(y, \theta)\right| \leqslant A|\theta|^{2 m}, \quad A>0 .
$$


Далее, пусть $y \in \overline{\Gamma_{i}}$. Без ограничения общности можем считать, что вблизи заданной точки $y$ кривая $\overline{\Gamma_{i}}$ определена уравнением $y_{2}=0$. Предположим, что для всех $i=1, \ldots, N$ система операторов $\left\{B_{i \mu 0}\left(y, D_{y}\right)\right\}_{\mu=1}^{m}$ удовлетворяет условию накрытия по отношению $\kappa$ оператору $\mathbf{P}\left(y, D_{y}\right)$. Другими словами, пусть многочлен

$$
B_{i \mu 0}^{\prime}(y, \tau) \equiv \sum_{\nu=1}^{m} b_{i \mu \nu}(y) \tau^{\nu-1} \equiv B_{i \mu 0}^{0}(y, 1, \tau)\left(\bmod \mathbf{M}^{+}(y, \tau)\right)
$$

есть остаток от деления $B_{i \mu 0}^{0}(y, 1, \tau)$ на $\mathbf{M}^{+}(y, \tau)$, где

$$
\mathbf{M}^{+}(y, \tau)=\prod_{\nu=1}^{m}\left(\tau-\tau_{\nu}^{+}(y)\right)
$$

$\tau_{1}^{+}(y), \ldots, \tau_{m}^{+}(y)$ - корни с положительной мнимой частью многочлена $\mathbf{P}^{0}(y, 1, \tau)$, причем $\mathbf{P}^{0}(y, 1, \tau), B_{i \mu 0}^{0}(y, 1, \tau)$ и $\mathbf{M}^{+}(y, \tau)$ рассматриваются как многочлены от $\tau$. Тогда условие накрытия означает, что

$$
d_{i}(y)=\operatorname{det}\left\|b_{i \mu \nu}(y)\right\|_{\mu, \nu=1}^{m} \neq 0 .
$$

Так как каждая из дуг $\overline{\Gamma_{i}}, i=1, \ldots, N,-$ компакт, то

$$
D=\min _{i=1, \ldots, N} \inf _{y \in \overline{\Gamma_{i}}}\left|d_{i}(y)\right|>0
$$

Подчеркнем, что нормальность операторов $B_{i \mu 0}\left(y, D_{y}\right)$ на дугах $\overline{\Gamma_{i}}$ не предполагается. Для целых $k \geqslant 0$ обозначим через $W^{k}(G)=W_{2}^{k}(G)$ пространство Соболева с нормой

$$
\|u\|_{W^{k}(G)}=\left(\sum_{|\alpha| \leqslant k} \int_{G}\left|D^{\alpha} u\right|^{2} d y\right)^{1 / 2}
$$

(при $k=0$ полагаем $\left.W^{0}(G)=L_{2}(G)\right)$. Для цельх $k \geqslant 1$ введем пространство $W^{k-1 / 2}(\Gamma)$ следов на гладкой кривой $\Gamma \subset \bar{G}$ с нормой

$$
\|\psi\|_{W^{k-1 / 2}(\Gamma)}=\inf \|u\|_{W^{k}(G)}, \quad u \in W^{k}(G):\left.u\right|_{\Gamma}=\psi
$$

Положим

$$
\mathbf{B}_{i \mu}^{0} u=\left.B_{i \mu 0}\left(y, D_{y}\right) u(y)\right|_{\Gamma_{i}} .
$$

Как отмечалось вьше, операторы $\mathbf{P}\left(y, D_{y}\right)$ и $\mathbf{B}_{i \mu}^{0}$ будут coответствовать “локальной" краевой задаче.

Определим операторы, соответствующие нелокальным условиям вблизи множества $\mathscr{K}$. Пусть $\Omega_{i s}, i=1, \ldots, N, s=1, \ldots, S_{i},-$ бесконечно дифференцируемые невырожденные преобразования, отображающие некоторую окрестность $\mathscr{O}_{i}$ кривой $\overline{\Gamma_{i} \cap \mathscr{O}_{\varepsilon}(\mathscr{K})}$ на множество $\Omega_{i s}\left(\mathscr{O}_{i}\right)$ так, что $\Omega_{i s}\left(\Gamma_{i} \cap \mathscr{O}_{\varepsilon}(\mathscr{K})\right) \subset G$ и

$$
\Omega_{i s}(g) \in \mathscr{K} \quad \text { для } g \in \overline{\Gamma_{i}} \cap \mathscr{K} .
$$


Здесь $\varepsilon>0, \mathscr{O}_{\varepsilon}(\mathscr{K})=\left\{y \in \mathbb{R}^{2}: \operatorname{dist}(y, \mathscr{K})<\varepsilon\right\}-\varepsilon$-окрестность множества $\mathscr{K}$. Таким образом, преобразования $\Omega_{i s}$ отображают дуги $\Gamma_{i} \cap \mathscr{O}_{\varepsilon}(\mathscr{K})$ строго внутрь области $G$, а их концевые точки $\overline{\Gamma_{i}} \cap \mathscr{K}$ в концевые точки.

Уточним структуру преобразований $\Omega_{i s}$ вблизи множества $\mathscr{K}$. Обозначим преобразование $\Omega_{i s}: \mathscr{O}_{i} \rightarrow \Omega_{i s}\left(\mathscr{O}_{i}\right)$ через $\Omega_{i s}^{+1}$, а через $\Omega_{i s}^{-1}: \Omega_{i s}\left(\mathscr{O}_{i}\right) \rightarrow \mathscr{O}_{i}$ преобразование, обратное к $\Omega_{i s}$. Назовем орбитой точки $g \in \mathscr{K}$ и обозначим $\operatorname{Orb}(g)$ множество всех точек вида $\Omega_{i_{q} s_{q}}^{ \pm 1}\left(\ldots \Omega_{i_{1} s_{1}}^{ \pm 1}(g)\right) \in \mathscr{K}, 1 \leqslant s_{j} \leqslant S_{i_{j}}, j=1, \ldots, q$, т.е. всех тех, которые можно получить, применяя к $g$ последовательно преобразования $\Omega_{i_{j} s_{j}}^{+1}$ или $\Omega_{i_{j} s_{j}}^{-1}$, отображаюшие точки множества $\mathscr{K}$ в $\mathscr{K}$.

Очевидно, для любых $g, g^{\prime} \in \mathscr{K}$ либо $\operatorname{Orb}(g)=\operatorname{Orb}\left(g^{\prime}\right)$, либо $\operatorname{Orb}(g) \cap \operatorname{Orb}\left(g^{\prime}\right)=\varnothing$. Далее будем считать, что множество $\mathscr{K}$ состоит из одной орбиты (все результаты без труда переносятся на общий случай, когда $\mathscr{K}$ состоит из конечного числа непересекающихся орбит). Очевидно, множество (орбита) $\mathscr{K}$ состоит из $N$ точек; обозначим их через $g_{j}, j=1, \ldots, N$.

Будем считать $\varepsilon$ настолько малым (см. ниже замечание 1.3 ), чтобы существовали окрестности $\mathscr{O}_{\varepsilon_{1}}\left(g_{j}\right)$ точек $g_{j} \in \mathscr{K}$ такие, что $\mathscr{O}_{\varepsilon_{1}}\left(g_{j}\right) \supset \mathscr{O}_{\varepsilon}\left(g_{j}\right)$ и

1) в окрестности $\mathscr{O}_{\varepsilon_{1}}\left(g_{j}\right)$ граница $\partial G$ совпадает с некоторым углом;

2) $\overline{\mathscr{O}_{\varepsilon_{1}}\left(g_{j}\right)} \cap \overline{\mathscr{O}_{\varepsilon_{1}}\left(g_{k}\right)}=\varnothing$ для любых $g_{j}, g_{k} \in \mathscr{K}, k \neq j$;

3) если $g_{j} \in \overline{\Gamma_{i}}$ и $\Omega_{i s}\left(g_{j}\right)=g_{k}$, то $\mathscr{O}_{\varepsilon}\left(g_{j}\right) \subset \mathscr{O}_{i}$ и $\Omega_{i s}\left(\mathscr{O}_{\varepsilon}\left(g_{j}\right)\right) \subset \mathscr{O}_{\varepsilon_{1}}\left(g_{k}\right)$.

Для каждой точки $g_{j} \in \overline{\Gamma_{i}} \cap \mathscr{K}$ зафиксируем преобразование $y \mapsto y^{\prime}\left(g_{j}\right)$, представляющее собой сдвиг на вектор $-\overrightarrow{O g_{j}}$ и поворот на некоторьй угол и переводящее множество $\mathscr{O}_{\varepsilon_{1}}\left(g_{j}\right)$ в окрестность нуля $\mathscr{O}_{\varepsilon_{1}}(0)$, a $G \cap \mathscr{O}_{\varepsilon_{1}}\left(g_{j}\right)$ и $\Gamma_{i} \cap \mathscr{O}_{\varepsilon_{1}}\left(g_{j}\right)$ соответственно в пересечение плоского угла $K_{j}=\left\{y \in \mathbb{R}^{2}: r>0,|\omega|<\omega_{j}\right\}$ с окрестностью $\mathscr{O}_{\varepsilon_{1}}(0)$ и пересечение стороны $\gamma_{j \sigma}=\left\{y \in \mathbb{R}^{2}: \omega=(-1)^{\sigma} \omega_{j}\right\}(\sigma=1$ или $\sigma=2)$ угла $K_{j} \mathrm{c}$ окрестностью $\mathscr{O}_{\varepsilon_{1}}(0)$. Здесь $(\omega, r)$ - полярные координаты точки $y, 0<\omega_{j}<\pi$.

УСловИЕ 1.1. Преобразованию $\Omega_{i s}(y), i=1, \ldots, N, s=1, \ldots, S_{i}$, при у $\in \mathscr{O}_{\varepsilon}\left(g_{j}\right)$, $g_{j} \in \overline{\Gamma_{i}} \cap \mathscr{K}$, в новой системе координат $\left\{y^{\prime}\right\}$ соответствует поворот и растяжение вектора $y^{\prime}$.

ЗАмЕчАнИЕ 1.1. Условие 1.1 совместно с условием $\Omega_{i s}\left(\Gamma_{i}\right) \subset G$ означает, что если $g \in \Omega_{i s}\left(\overline{\Gamma_{i}} \cap \mathscr{K}\right) \cap \overline{\Gamma_{j}} \cap \mathscr{K} \neq \varnothing$, то кривые $\Omega_{i s}\left(\overline{\Gamma_{i}}\right)$ и $\overline{\Gamma_{j}}$ пересекаются в точке $g$ под ненулевым углом.

Выберем число $\varepsilon_{0}, 0<\varepsilon_{0} \leqslant \varepsilon$, удовлетворяющее следующему условию: если $g_{j} \in \overline{\Gamma_{i}}$ и $\Omega_{i s}\left(g_{j}\right)=g_{k}$, то $\mathscr{O}_{\varepsilon_{0}}\left(g_{k}\right) \subset \Omega_{i s}\left(\mathscr{O}_{\varepsilon}\left(g_{j}\right)\right)$. Рассмотрим функцию $\zeta \in C^{\infty}\left(\mathbb{R}^{2}\right)$ такую, что

$$
\zeta(y)=1, \quad y \in \mathscr{O}_{\varepsilon_{0} / 2}(\mathscr{K}), \quad \operatorname{supp} \zeta \subset \mathscr{O}_{\varepsilon_{0}}(\mathscr{K})
$$

Введем нелокальные операторы $\mathbf{B}_{i \mu}^{1}$ по формуле

$$
\begin{gathered}
\mathbf{B}_{i \mu}^{1} u=\sum_{s=1}^{S_{i}}\left(B_{i \mu s}\left(y, D_{y}\right)(\zeta u)\right)\left(\Omega_{i s}(y)\right), \quad y \in \Gamma_{i} \cap \mathscr{O}_{\varepsilon}(\mathscr{K}), \\
\mathbf{B}_{i \mu}^{1} u=0, \quad y \in \Gamma_{i} \backslash\left(\Gamma_{i} \cap \mathscr{O}_{\varepsilon}(\mathscr{K})\right)
\end{gathered}
$$


(запись $\left(B_{i \mu s}\left(y, D_{y}\right) u\right)\left(\Omega_{i s}(y)\right)$ означает, что выражение $B_{i \mu s}\left(x, D_{x}\right) u(x)$ берется при значении аргумента $\left.x=\Omega_{i s}(y)\right)$. Поскольку $\mathbf{B}_{i \mu}^{1} u=0$, если

$$
\operatorname{supp} u \subset \bar{G} \backslash \overline{\mathscr{O}_{\varepsilon_{0}}(\mathscr{K})},
$$

будем говорить, что операторы $\mathbf{B}_{i \mu}^{1}$ соответствуют нелокальным. слагаемым с носителем вблизи множества $\mathscr{K}$.

Для $\rho>0$ обозначим $G_{\rho}=\{y \in G: \operatorname{dist}(y, \partial G)>\rho\}$. Рассмотрим операторы $\mathbf{B}_{i \mu}^{2}$, удовлетворяющие следующему условию (ср. [13], [18], [22]).

УСЛОВИЕ 1.2. Существуют числа $\varkappa_{1}>\varkappa_{2}>0 u \rho>0$ такие, что для всех

$$
u \in W^{2 m}\left(G \backslash \overline{\mathscr{O}_{\varkappa_{1}}(\mathscr{K})}\right) \cap W^{2 m}\left(G_{\rho}\right)
$$

выполнены неравенства

$$
\begin{aligned}
\left\|\mathbf{B}_{i \mu}^{2} u\right\|_{W^{2 m-m_{i \mu}-1 / 2}\left(\Gamma_{i}\right)} & \leqslant c_{1}\|u\|_{W^{2 m}\left(G \backslash \overline{\mathscr{O}_{\varkappa_{1}}(\mathscr{K})}\right)}, \\
\left\|\mathbf{B}_{i \mu}^{2} u\right\|_{W^{2 m-m_{i \mu}-1 / 2}\left(\Gamma_{i} \backslash \overline{\mathscr{O}_{\varkappa_{2}}(\mathscr{K})}\right)} & \leqslant c_{2}\|u\|_{W^{2 m}\left(G_{\rho}\right)}
\end{aligned}
$$

здесъ $i=1, \ldots, N, \mu=1, \ldots, m, c_{1}, c_{2}>0$.

Из неравенства (1.6), в частности, следует, что $\mathbf{B}_{i \mu}^{2} u=0$, если $\operatorname{supp} u \subset \mathscr{O}_{\varkappa_{1}}(\mathscr{K})$. Поэтому будем говорить, что операторы $\mathbf{B}_{i \mu}^{2}$ соответствуют нелокальным слагаемым с носителем вне множества $\mathscr{K}$.

Условия 1.1 и 1.2 будем предполагать выполненньпи на протяжении всей статьи.

Рассмотрим нелокальную краевую задачу

$$
\begin{array}{ll}
\mathbf{P}\left(y, D_{y}\right) u=f_{0}(y), & y \in G, \\
\mathbf{B}_{i \mu} u \equiv \mathbf{B}_{i \mu}^{0} u+\mathbf{B}_{i \mu}^{1} u+\mathbf{B}_{i \mu}^{2} u=0, & y \in \Gamma_{i}, \quad i=1, \ldots, N, \quad \mu=1, \ldots, m,
\end{array}
$$

где $f_{0} \in L_{2}(G)$. Введем пространство $W_{B}^{m}(G)$, состоящее из функций $u \in W^{m}(G)$, удовлетворяющих однородньм нелокальным условиям (1.9): $\mathbf{B}_{i \mu} u=0$.

Рассмотрим неограниченный оператор $\mathbf{P}: \operatorname{Dom}(\mathbf{P}) \subset L_{2}(G) \rightarrow L_{2}(G)$, действующий в пространстве распределений $\mathscr{D}^{\prime}(G)$ по формуле

$$
\mathbf{P} u=\mathbf{P}\left(y, D_{y}\right) u, \quad u \in \operatorname{Dom}(\mathbf{P})=\left\{u \in W_{B}^{m}(G): \mathbf{P}\left(y, D_{y}\right) u \in L_{2}(G)\right\} .
$$

ОПРЕДЕЛЕНИЕ 1.1. Функция $u$ называется обобщенным решением задачи (1.8), (1.9) с правой частью $f_{0} \in L_{2}(G)$, если $u \in \operatorname{Dom}(\mathbf{P})$ и $\mathbf{P} u=f_{0}$.

Можно также дать другое, эквивалентное, определение обобщенного решения. Для этого запишем оператор $\mathbf{P}\left(y, D_{y}\right)$ в дивергентной форме:

$$
\mathbf{P}\left(y, D_{y}\right)=\sum_{0 \leqslant|\xi|,|\beta| \leqslant m} D^{\beta} p_{\xi \beta}(y) D^{\xi},
$$

где $p_{\xi \beta}-$ бесконечно дифференцируемые функции.

Для всякого множества $X \in \mathbb{R}^{2}$, имеющего непустую внутренность, будем обозначать через $C_{0}^{\infty}(X)$ множество бесконечно дифференцируемых в $\bar{X}$ функций с компактными носителями, содержащимися в $X$. 
ОПРЕДЕЛЕНИЕ 1.2. Функция $u$ называется обобщенным решением задачи (1.8), (1.9) с правой частью $f_{0} \in L_{2}(G)$, если $u \in W_{B}^{m}(G)$ и для всех $v \in C_{0}^{\infty}(G)$ вьполнено интегральное тождество

$$
\sum_{0 \leqslant|\xi|,|\beta| \leqslant m} \int_{G} p_{\xi \beta}(y) D^{\xi} u \overline{D^{\beta} v} d y=\int_{G} f_{0} \bar{v} d y .
$$

ЗАмЕчАниЕ 1.2. Обобщенные решения априори принадлежат пространству $W^{m}(G)$, тогда как условие 1.2 сформулировано для функций, принадлежащих пространству $W^{2 m}$ внутри области и вблизи гладкой части границы. Такая формулировка оправдана тем, что всякое обобщенное решение в действительности принадлежит пространству $W^{2 m}$ вне сколь угодно малой окрестности множества $\mathscr{K}$ (см. ниже лемму 2.1).

ЗАМЕчАНИЕ 1.3. Выше мы предположили, что число $\varepsilon$ достаточно мало (тогда как $\varkappa_{1}, \varkappa_{2}, \rho$ могут быть произвольными). Покажем, что это не ограничивает общности. Пусть имеется число $\widehat{\varepsilon}, 0<\widehat{\varepsilon}<\varepsilon$. Выберем число $\widehat{\varepsilon}_{0}, 0<\widehat{\varepsilon}_{0} \leqslant \widehat{\varepsilon}$, удовлетворяющее следующему условию: если $g_{j} \in \overline{\Gamma_{i}}$ и $\Omega_{i s}\left(g_{j}\right)=g_{k}$, то $\mathscr{O}_{\widehat{\varepsilon}_{0}}\left(g_{k}\right) \subset \Omega_{i s}\left(\mathscr{O}_{\widehat{\varepsilon}}\left(g_{j}\right)\right)$. Рассмотрим функцию $\widehat{\zeta} \in C^{\infty}\left(\mathbb{R}^{2}\right)$ такую, что $\widehat{\zeta}(y)=1$ при $y \in \mathscr{O}_{\widehat{\varepsilon}_{0} / 2}(\mathscr{K})$ и $\operatorname{supp} \widehat{\zeta} \subset \mathscr{O}_{\widehat{\varepsilon}_{0}}(\mathscr{K})$. Введем операторы $\mathbf{B}_{i \mu}^{1}$ следуюшим образом:

$$
\begin{array}{ll}
\mathbf{B}_{i \mu}^{1} u=\sum_{s=1}^{S_{i}}\left(B_{i \mu s}\left(y, D_{y}\right)(\zeta u)\right)\left(\Omega_{i s}(y)\right) & \text { при } y \in \Gamma_{i} \cap \mathscr{O}_{\varepsilon}(\mathscr{K}), \\
\mathbf{B}_{i \mu}^{1} u=0 & \text { при } y \in \Gamma_{i} \backslash\left(\Gamma_{i} \cap \mathscr{O}_{\varepsilon}(\mathscr{K})\right) .
\end{array}
$$

Очевидно,

$$
\mathbf{B}_{i \mu}^{0}+\mathbf{B}_{i \mu}^{1}+\mathbf{B}_{i \mu}^{2}=\mathbf{B}_{i \mu}^{0}+\widehat{\mathbf{B}}_{i \mu}^{1}+\widehat{\mathbf{B}}_{i \mu}^{2},
$$

где $\widehat{\mathbf{B}}_{i \mu}^{2}=\mathbf{B}_{i \mu}^{1}-\widehat{\mathbf{B}}_{i \mu}^{1}+\mathbf{B}_{i \mu}^{2}$. Так как $\mathbf{B}_{i \mu}^{1} u-\widehat{\mathbf{B}}_{i \mu}^{1} u=0$ вблизи множества $\mathscr{K}$, то оператор $\mathbf{B}_{i \mu}^{1}-\widehat{\mathbf{B}}_{i \mu}^{1}$ удовлетворяет при соответствующих $\varkappa_{1}, \varkappa_{2}, \rho$ условию 1.2 (более подробно см. $[22, \S 1])$. Таким образом, число $\varepsilon$ всегда может быть выбрано настолько мальм, насколько нам требуется. При этом, однако, следует иметь в виду, что оператор $\mathbf{B}_{i \mu}^{2}$ и значения $\varkappa_{1}, \varkappa_{2}, \rho$ могут измениться.

1.2. Пример нелокальной задачи. Модельньм для нас может служить следующий пример.

ПРИмеР 1.1. Пусть операторы $\mathbf{P}\left(y, D_{y}\right)$ и $B_{i \mu s}\left(y, D_{y}\right)$ те же, что и вьше. Пусть $\Omega_{i s}, i=1, \ldots, N, s=1, \ldots, S_{i},-$ бесконечно дифференцируемые невырожденные преобразования, отображающие некоторую окрестность $\mathscr{O}_{i}\left(\right.$ всей) кривой $\Gamma_{i}$ на множество $\Omega_{i s}\left(\mathscr{O}_{i}\right)$ так, что $\Omega_{i s}\left(\Gamma_{i}\right) \subset G$. Рассмотрим нелокальную задачу

$$
\begin{gathered}
\mathbf{P}\left(y, D_{y}\right) u=f_{0}(y), \quad y \in G \\
\left.B_{i \mu 0}\left(y, D_{y}\right) u(y)\right|_{\Gamma_{i}}+\left.\sum_{s=1}^{S_{i}}\left(B_{i \mu s}\left(y, D_{y}\right) u\right)\left(\Omega_{i s}(y)\right)\right|_{\Gamma_{i}}=0 \\
y \in \Gamma_{i}, \quad i=1, \ldots, N, \quad \mu=1, \ldots, m .
\end{gathered}
$$


Подчеркнем: изначально мы не предполагаем, что преобразования $\Omega_{\text {is }}$ удовлетворяют условию (1.4); однако далее мы представим нелокальные операторы в виде суммы операторов $\mathbf{B}_{i \mu}^{0}, \mathbf{B}_{i \mu}^{1} u \mathbf{B}_{i \mu}^{2}$, причем преобразования, которые войдут в определение операторов $\mathbf{B}_{i \mu}^{1}$, будут удовлетворять условию (1.4). Выберем для этого $\varepsilon$ настолько малым, чтобы для любой точки $g \in \mathscr{K}$ множество $\overline{\mathscr{O}_{\varepsilon}(g)}$ пересекалось с дугой $\overline{\Omega_{i s}\left(\Gamma_{i}\right)}$ только, если $g \in \overline{\Omega_{i s}\left(\Gamma_{i}\right)}$. Если для $g \in \overline{\Gamma_{i}} \cap \mathscr{K}$ выполнено $\Omega_{i s}(g) \in \mathscr{K}$, то наложим на преобразование $\Omega_{i s}(y)$ при $y \in \mathscr{O}_{\varepsilon}(g)$ условие 1.1.

ЗАмЕчАниЕ 1.4. Согласно замечанию 1.1 условие 1.1 есть ограничение на геометрическую структуру носителя нелокальных членов вблизи множества $\mathscr{K}$. Если же $\Omega_{i s}\left(\overline{\Gamma_{i}} \cap \mathscr{K}\right) \subset \partial G \backslash \mathscr{K}$, то на характер подхода кривой $\Omega_{i s}\left(\overline{\Gamma_{i}}\right)$ к границе $\partial G$ (так же, как и в работах [13], [17]) никаких ограничений не налагается.

Обозначим через $\zeta \in C^{\infty}\left(\mathbb{R}^{2}\right)$ функцию, удовлетворяющую соотношениям (1.5), и введем операторы

$$
\begin{gathered}
\mathbf{B}_{i \mu}^{0} u=\left.B_{i \mu 0}\left(y, D_{y}\right) u(y)\right|_{\Gamma_{i}}, \quad \mathbf{B}_{i \mu}^{1} u=\left.\sum_{s=1}^{S_{i}}\left(B_{i \mu s}\left(y, D_{y}\right)(\zeta u)\right)\left(\Omega_{i s}(y)\right)\right|_{\Gamma_{i}}, \\
\mathbf{B}_{i \mu}^{2} u=\left.\sum_{s=1}^{S_{i}}\left(B_{i \mu s}\left(y, D_{y}\right)((1-\zeta) u)\right)\left(\Omega_{i s}(y)\right)\right|_{\Gamma_{i}}
\end{gathered}
$$

(см. рис. 1.1, 1.2).

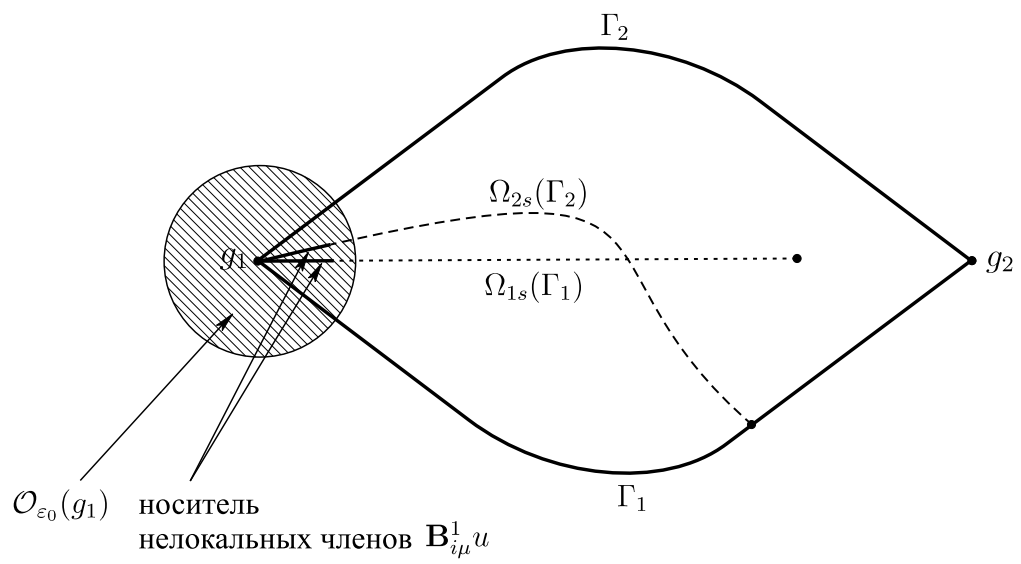

Рис. 1.1

Так как носитель функции $\zeta$ сосредоточен вблизи множества $\mathscr{K}$, можно считать, что преобразования $\Omega_{i s}$, фигурируюшие в определении оператора $\mathbf{B}_{i \mu}^{1}$, заданы в некоторой окрестности множества $\mathscr{K}$ и удовлетворяют условию (1.4). Кроме того, согласно [22, п. 1.2] оператор $\mathbf{B}_{i \mu}^{2}$ удовлетворяет условию 1.2. Следовательно, задача (1.10), (1.11) представима в виде (1.8), (1.9).

1.3. Нелокальные задачи вблизи множества $\mathscr{K}$. При изучении задачи (1.8), (1.9) особое внимание следует уделять поведению решений в окрестности множества $\mathscr{K}$ точек сопряжения нелокальных условий. Вьпишем соответствуюшие модельные задачи. 


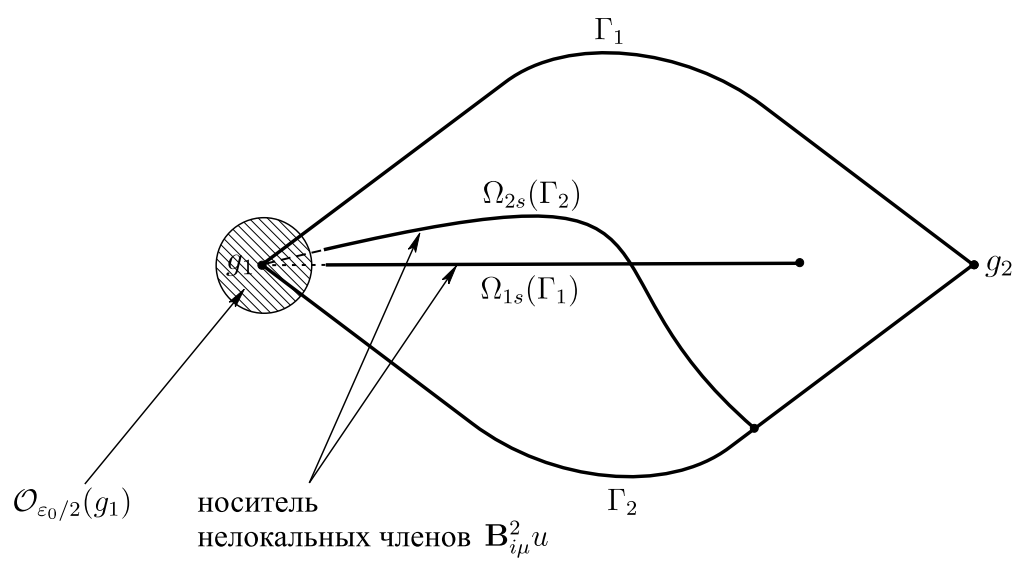

Рис. 1.2

Обозначим $u(y)$ при $y \in \mathscr{O}_{\varepsilon_{1}}\left(g_{j}\right)$ через $u_{j}(y)$. Если $g_{j} \in \overline{\Gamma_{i}}, y \in \mathscr{O}_{\varepsilon}\left(g_{j}\right), \Omega_{i s}(y) \in$ $\mathscr{O}_{\varepsilon_{1}}\left(g_{k}\right)$, то обозначим $u\left(\Omega_{i s}(y)\right)$ через $u_{k}\left(\Omega_{i s}(y)\right)$. Тогда нелокальная задача $(1.8),(1.9)$ в $\varepsilon$-окрестности множества (орбиты) $\mathscr{K}$ примет вид

$$
\begin{aligned}
& \mathbf{P}\left(y, D_{y}\right) u_{j}=f_{0}(y), \quad y \in \mathscr{O}_{\varepsilon}\left(g_{j}\right) \cap G, \\
& \left.B_{i \mu 0}\left(y, D_{y}\right) u_{j}(y)\right|_{\mathscr{O}_{\varepsilon}\left(g_{j}\right) \cap \Gamma_{i}}+\left.\sum_{s=1}^{S_{i}}\left(B_{i \mu s}\left(y, D_{y}\right)\left(\zeta u_{k}\right)\right)\left(\Omega_{i s}(y)\right)\right|_{\mathscr{O}_{\varepsilon}\left(g_{j}\right) \cap \Gamma_{i}}=f_{i \mu}(y), \\
& \quad y \in \mathscr{O}_{\varepsilon}\left(g_{j}\right) \cap \Gamma_{i}, \quad i \in\left\{1 \leqslant i \leqslant N: g_{j} \in \overline{\Gamma_{i}}\right\}, \quad j=1, \ldots, N, \quad \mu=1, \ldots, m,
\end{aligned}
$$

где $f_{i \mu}=-\mathbf{B}_{i \mu}^{2} u$.

Пусть $y \mapsto y^{\prime}\left(g_{j}\right)$ - описанные в п. 1.1 преобразования координат. Обозначим $K_{j}^{\varepsilon}=$ $K_{j} \cap \mathscr{O}_{\varepsilon}(0), \gamma_{j \sigma}^{\varepsilon}=\gamma_{j \sigma} \cap \mathscr{O}_{\varepsilon}(0)$, введем функции

$$
U_{j}\left(y^{\prime}\right)=u_{j}\left(y\left(y^{\prime}\right)\right), \quad f_{j}\left(y^{\prime}\right)=f_{0}\left(y\left(y^{\prime}\right)\right), \quad y^{\prime} \in K_{j}^{\varepsilon}, \quad f_{j \sigma \mu}\left(y^{\prime}\right)=f_{i \mu}\left(y\left(y^{\prime}\right)\right), \quad y^{\prime} \in \gamma_{j \sigma}^{\varepsilon},
$$

где $\sigma=1(\sigma=2)$, если преобразование $y \mapsto y^{\prime}\left(g_{j}\right)$ переводит $\Gamma_{i}$ в сторону $\gamma_{j 1}\left(\gamma_{j 2}\right)$ угла $K_{j}$, и переобозначим $y^{\prime}$ через $y$. Тогда в силу условия 1.1 задача $(1.8),(1.9)$ примет вид

$$
\begin{array}{ll}
\mathbf{P}_{j}\left(y, D_{y}\right) U_{j}=f_{j}(y), & y \in K_{j}^{\varepsilon}, \\
\sum_{k, s}\left(B_{j \sigma \mu k s}\left(y, D_{y}\right) U_{k}\right)\left(\mathscr{G}_{j \sigma k s} y\right)=f_{j \sigma \mu}(y), & y \in \gamma_{j \sigma}^{\varepsilon} .
\end{array}
$$

Здесь (и далее, если не оговорено противное) $j, k=1, \ldots, N, \sigma=1,2, \mu=1, \ldots, m$, $s=0, \ldots, S_{j \sigma k} ; \mathbf{P}_{j}\left(y, D_{y}\right)$ и $B_{j \sigma \mu k s}\left(y, D_{y}\right)$ - операторы порядков $2 m$ и $m_{j \sigma \mu}, m_{j \sigma \mu} \leqslant$ $m-1$, соответственно с переменными коэффициентами класса $C^{\infty}$ :

$$
\mathbf{P}_{j}\left(y, D_{y}\right)=\sum_{|\alpha| \leqslant 2 m} p_{j \alpha}(y) D_{y}^{\alpha}, \quad B_{j \sigma \mu k s}\left(y, D_{y}\right)=\sum_{|\alpha| \leqslant m_{j \sigma \mu}} b_{j \sigma \mu k s \alpha}(y) D_{y}^{\alpha} ;
$$

$\mathscr{G}_{j \sigma k s}$ - оператор поворота на угол $\omega_{j \sigma k s}$ и растяжения в $\chi_{j \sigma k s}>0$ раз в плоскости $\{y\}$, причем $\left|(-1)^{\sigma} b_{j}+\omega_{j \sigma k s}\right|<b_{k}$, если $(k, s) \neq(j, 0)$ (см. замечание 1.1$)$, и $\omega_{j \sigma j 0}=0$, $\chi_{j \sigma j 0}=1$ (т.е. $\mathscr{G}_{j \sigma j 0} y \equiv y$ ). 
2. Фредгольмова разрешимость нелокальных задач. Сформулируем основной результат, доказательству которого будет посвящен этот раздел.

TЕорема 2.1. Пусть оператор $\mathbf{P}\left(y, D_{y}\right)$ собственно әллиптичен на $\bar{G}$ и для всех $i=1, \ldots, N$ система операторов $\left\{B_{i \mu 0}\left(y, D_{y}\right)\right\}_{\mu=1}^{m}$ удовлетворяет условию накрытия по отношению $\kappa \mathbf{P}\left(y, D_{y}\right)$ на дуге $\overline{\Gamma_{i}}$. Пусть выполнены условия 1.1

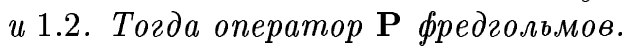

ЗАмЕчАниЕ 2.1. Задаче (1.8), (1.9) можно сопоставить ограниченньй оператор, действуюший из $W^{2 m}(G)$ в $L_{2}(G)$. Такой оператор изучен в [22], [23]: показано, что в отличие от рассматриваемого случая на его фредгольмовость влияют спектральные свойства некоторых вспомогательных нелокальных задач с параметром и выполнение определенных алгебраических соотношений между операторами $\mathbf{P}\left(y, D_{y}\right), \mathbf{B}_{i \mu}^{0}$ и $\mathbf{B}_{i \mu}^{1}$ в точках множества $\mathscr{K}$.

2.1. Конечномерность ядра. В этом пункте мы установим конечномерность ядра оператора Р. Для этого предварительно исследуем гладкость обобщенных решений задачи $(1.8),(1.9)$ сначала вне некоторой окрестности множества $\mathscr{K}$, а затем вблизи множества $\mathscr{K}$. Следующая лемма является обобщением части 1 теоремы 5 в [24].

ЛЕмма 2.1. Пусть выполнено условие 1.2. Пусть $и \in W^{m}(G)$ - обобщенное решение задачи (1.8), (1.9) с правой частью $f_{0} \in L_{2}(G)$. Тогда

$$
u \in W^{2 m}\left(G \backslash \overline{\mathscr{O}_{\delta}(\mathscr{K})}\right) \quad \text { при любом } \delta>0 .
$$

ДокАЗАТЕЛЬСтво. 1) Обозначим через $W_{\text {loc }}^{k}(G)$ пространство распределений $v$ на $G$ таких, что $\psi v \in W^{k}(G)$ для всех $\psi \in C_{0}^{\infty}(G)$. По теореме 3.2 из [25, гл. 2] имеем

$$
u \in W_{\operatorname{loc}}^{2 m}(G)
$$

Отсюда и из оценки (1.7) следует, что

$$
\mathbf{B}_{i \mu}^{2} u \in W^{2 m-m_{i \mu}-1 / 2}\left(\Gamma_{i} \backslash \overline{\mathscr{O}_{\varkappa_{2}}(\mathscr{K})}\right) .
$$

Зафиксируем произвольную точку $g \in \Gamma_{i} \backslash \overline{\mathscr{O}_{\varkappa_{2}}(\mathscr{K})}$. Выберем $\delta>0$ такое, что

$$
\overline{\mathscr{O}_{\delta}(g) \cap \Gamma_{i}} \subset \Gamma_{i} \backslash \overline{\mathscr{O}_{\varkappa_{2}}(\mathscr{K})}
$$

Тогда в окрестности $\mathscr{O}_{\delta}(g)$ функция $u$ есть решение “локальной” задачи

$$
\begin{array}{ll}
\mathbf{P}\left(y, D_{y}\right) u=f_{0}(y), & y \in \mathscr{O}_{\delta}(g) \cap G, \\
B_{i \mu 0}\left(y, D_{y}\right) u=f_{i \mu}^{\prime}(y), & y \in \mathscr{O}_{\delta}(g) \cap \Gamma_{i}, \quad \mu=1, \ldots, m,
\end{array}
$$

где $f_{i \mu}^{\prime}(y)=-\mathbf{B}_{i \mu}^{1} u(y)-\mathbf{B}_{i \mu}^{2} u(y), y \in \mathscr{O}_{\delta}(g) \cap \Gamma_{i}$. В силу соотношений (2.2)-(2.4) и определения оператора $\mathbf{B}_{i \mu}^{1}$ имеем $f_{i \mu}^{\prime} \in W^{2 m-m_{i \mu}-1 / 2}\left(\mathscr{O}_{\delta}(g) \cap \Gamma_{i}\right)$. 
Применяя к задаче $(2.5),(2.6)$ теорему 8.2 из $[25, \text { гл. } 2]^{1}$, получим, что

$$
u \in W^{2 m}\left(\mathscr{O}_{\delta / 2}(g) \cap G\right)
$$

Используя метод разбиения единищы, из (2.2) и (2.7) выводим

$$
u \in W^{2 m}\left(G \backslash \overline{\mathscr{O}_{\varkappa_{1}}(\mathscr{K})}\right) .
$$

2) Из включения (2.8) и неравенства (1.6) следует, что

$$
\mathbf{B}_{i \mu}^{2} u \in W^{2 m-m_{i \mu}-1 / 2}\left(\Gamma_{i}\right)
$$

Учитывая (2.9), мы можем повторить рассуждения п. 1) доказательства для произвольной точки $g \in \Gamma_{i}$ и $\delta>0$ такого, что

$$
\overline{\mathscr{O}_{\delta}(g) \cap \Gamma_{i}} \subset \Gamma_{i}
$$

В результате получим включение (2.7), справедливое уже для произвольной точки $g \in \Gamma_{i}$. Отсюда и из (2.2), используя метод разбиения единицы, выводим (2.1).

Теперь изучим гладкость решений задачи (1.8), (1.9) в окрестности множества $\mathscr{K}$. Так как вблизи множества $\mathscr{K}$ обобщенные решения могут иметь степенные особенности [13], то решения естественно рассматривать в соответствующих весовых пространствах. Введем эти пространства.

Пусть либо $Q=\left\{y \in \mathbb{R}^{2}: r>0,|\omega|<b\right\}$, либо $Q=\left\{y \in \mathbb{R}^{2}: 0<r<d,|\omega|<b\right\}$, $0<b<\pi, d>0$, либо $Q=G$; в первом и втором случаях обозначим через $\mathscr{M}$ множество $\{0\}$, а в третьем случае-множество $\mathscr{K}$. Введем пространство $H_{a}^{k}(Q)$ как пополнение множества $C_{0}^{\infty}(\bar{Q} \backslash \mathscr{M})$ по норме

$$
\|w\|_{H_{a}^{k}(Q)}=\left(\sum_{|\alpha| \leqslant k} \int_{Q} \rho^{2(a-k+|\alpha|)}\left|D_{y}^{\alpha} w\right|^{2} d y\right)^{1 / 2}
$$

где $a \in \mathbb{R}, k \geqslant 0$ - целое, $\rho=\rho(y)=\operatorname{dist}(y, \mathscr{M})$. Через $H_{a}^{k-1 / 2}(\gamma)$ при $k \geqslant 1$ обозначим пространство следов на гладкой кривой $\gamma \subset \bar{Q}$ с нормой

$$
\|\psi\|_{H_{a}^{k-1 / 2}(\gamma)}=\inf \|w\|_{H_{a}^{k}(Q)}, \quad w \in H_{a}^{k}(Q):\left.w\right|_{\gamma}=\psi
$$

Пусть $u$ - обобшенное решение задачи $(1.8),(1.9)$, a $U_{j}\left(y^{\prime}\right)=u_{j}\left(y\left(y^{\prime}\right)\right), j=1, \ldots, N,-$ функции, соответствующие множеству (орбите) $\mathscr{K}$ и удовлетворяющие задаче (1.12), (1.13) с правой частью $\left\{f_{j}, f_{j \sigma \mu}\right\}$ (см. п. 1.3).

\footnotetext{
${ }^{1} \mathrm{~B}$ теореме 8.2 из [25, гл. 2] дополнительно предполагается, что операторы $B_{i \mu 0}\left(y, D_{y}\right)$ нормальны на $\Gamma_{i}$, а их порядки различны. Однако нетрудно проверить, что упомянутая теорема верна и без этого предположения (см. [25, гл. 2, п. 8.3]).
} 
Положим $d_{1}=\min \left\{\chi_{j \sigma k s}, 1\right\} / 2, d_{2}=2 \max \left\{\chi_{j \sigma k s}, 1\right\}$. Будем считать $\varepsilon$ настолько малым, что $d_{2} \varepsilon<\varepsilon_{1}$. Тогда в силу леммы 2.1

$$
U_{j} \in W^{2 m}\left(K_{j}^{d_{2} \varepsilon} \cap\{|y|>\delta\}\right) \quad \text { при любом } \delta>0 .
$$

Далее, из включения $U_{j} \in W^{m}\left(K_{j}^{d_{2} \varepsilon}\right)$ и из [26, лемма 5.2] следует, что

$$
U_{j} \in H_{a-m}^{m}\left(K_{j}^{d_{2} \varepsilon}\right) \subset H_{a-2 m}^{0}\left(K_{j}^{d_{2} \varepsilon}\right), \quad a>2 m-1 .
$$

Наконец, $f_{j} \in L_{2}\left(K_{j}^{\varepsilon}\right)$ и в силу леммы 2.1 и оценки $(1.6) f_{j \sigma \mu} \in W^{2 m-m_{j \sigma \mu}-1 / 2}\left(\gamma_{j \sigma}^{\varepsilon}\right)$. Следовательно, согласно [26, лемма 5.2]

$$
f_{j} \in H_{a}^{0}\left(K_{j}^{\varepsilon}\right), \quad f_{j \sigma \mu} \in H_{a}^{2 m-m_{j \sigma \mu}-1 / 2}\left(\gamma_{j \sigma}^{\varepsilon}\right), \quad a>2 m-1 .
$$

При помощи следующих двух лемм мы покажем, что из соотношений (2.11)-(2.13) вытекает включение $U_{j} \in H_{a}^{2 m}\left(K_{j}^{\varepsilon / d_{2}^{3}}\right)$.

Обозначим $K_{j q}=K_{j} \cap\left\{\varepsilon d_{2}^{-3} d_{1}^{4-q} / 2<|y|<\varepsilon d_{2}^{-3} d_{2}^{4-q}\right\}$, где $q=0, \ldots, 4$.

Лемма 2.2. Пусть выполнено условие 1.1. Тогда для всех $U \in \prod_{j} W^{2 m}\left(K_{j 0}\right)$ справедлива оценка

$$
\begin{aligned}
\sum_{j}\left\|U_{j}\right\|_{W^{2 m}\left(K_{j 4}\right)} \leqslant c & \sum_{j}\left\{\left\|\mathbf{P}_{j}\left(y, D_{y}\right) U_{j}\right\|_{L_{2}\left(K_{j 1}\right)}\right. \\
& +\sum_{\sigma, \mu}\left\|\left.\mathbf{B}_{j \sigma \mu}\left(y, D_{y}\right) U\right|_{\gamma_{j \sigma} \cap \overline{K_{j 1}}}\right\|_{W^{2 m-m_{j \sigma \mu}-1 / 2}\left(\gamma_{j \sigma} \cap \overline{K_{j 1}}\right)} \\
& \left.+\left\|U_{j}\right\|_{L_{2}\left(K_{j 1}\right)}\right\}
\end{aligned}
$$

әде $c>0$ не зависит от $U$.

ДоКАЗАТЕЛЬСТво. Из общей теории эллиптических уравнений следует, что

$$
\begin{aligned}
\left\|U_{j}\right\|_{W^{2 m}\left(K_{j 4}\right)} \leqslant & k_{1}\left(\left\|\mathbf{P}_{j}\left(y, D_{y}\right) U_{j}\right\|_{L_{2}\left(K_{j 3}\right)}\right. \\
& +\sum_{\sigma, \mu}\left\|\left.B_{j \sigma \mu j 0}\left(y, D_{y}\right) U_{j}\right|_{\gamma_{j \sigma} \cap \overline{K_{j 3}}}\right\|_{W^{2 m-m_{j \sigma \mu}-1 / 2}\left(\gamma_{j \sigma} \cap \overline{K_{j 3}}\right)} \\
& \left.+\left\|U_{j}\right\|_{L_{2}\left(K_{j 3}\right)}\right) .
\end{aligned}
$$

Пусть $(k, s) \neq(j, 0)$. Так как при этом $\mathscr{G}_{j \sigma k s}\left(\gamma_{j \sigma}\right) \cap \overline{K_{k 2}}$ лежит строго внутри области $K_{k 1}$, используя непрерьвность оператора следа в пространствах Соболева, аналогично (2.15) получаем

$$
\begin{aligned}
& \left\|\left.B_{j \sigma \mu k s}\left(y, D_{y}\right) U_{k}\left(\mathscr{G}_{j \sigma k s} y\right)\right|_{\gamma_{j \sigma} \cap \overline{K_{j 3}}}\right\|_{W^{2 m-m_{j \sigma \mu}-1 / 2}\left(\gamma_{j \sigma} \cap \overline{K_{j 3}}\right)} \\
& \quad \leqslant k_{2}\left\|\left.B_{j \sigma \mu k s}\left(y, D_{y}\right) U_{k}\right|_{\mathscr{G}_{j \sigma k s}\left(\gamma_{j \sigma}\right) \cap \overline{K_{k 2}}}\right\|_{W^{2 m-m_{j \sigma \mu}-1 / 2}\left(\mathscr{G}_{j \sigma k s}\left(\gamma_{j \sigma}\right) \cap \overline{K_{k 2}}\right)} \\
& \quad \leqslant k_{3}\left(\left\|\mathbf{P}_{j}\left(y, D_{y}\right) U_{k}\right\|_{L_{2}\left(K_{k 1}\right)}+\left\|U_{k}\right\|_{L_{2}\left(K_{k 1}\right)}\right) .
\end{aligned}
$$

Из оценок (2.15) и (2.16) вытекает (2.14). 
ЗАмЕчание 2.2 . Пусть нормы в $C^{0}\left(\overline{K_{j 1}}\right)$ коэффициентов $p_{j \alpha}$ операторов $\mathbf{P}_{j}\left(y, D_{y}\right)$ и нормы в $C^{2 m-m_{j \sigma \mu}}\left(\overline{K_{j 0}}\right)$ коэффициентов $b_{j \sigma \mu k s \alpha}$ операторов $B_{j \sigma \mu k s}\left(y, D_{y}\right)$ ограничены константой $C$. Пусть той же константой $C$ ограничены нормы в $C^{1}\left(\overline{K_{j 1}}\right)$ коэффициентов $p_{j \alpha},|\alpha|=2 m$, при старших производных в операторах $\mathbf{P}_{j}\left(y, D_{y}\right)$. Тогда константа $c$ в неравенстве (2.14) зависит только от $C$, от константы $A$ в (1.1) и от константы $D$ в (1.2).

ЛЕмма 2.3. Пусть выполнено условие 1.1. Предположим, что функиия $U$ удовлетворяет соотношениям (2.11), (2.12) и является решением задачи (1.12), (1.13) с правой частью $\left\{f_{j}, f_{j \sigma \mu}\right\}$, удовлетворяющей соотношениям (2.13). Тогда $U \in$ $\prod_{j} H_{a}^{2 m}\left(K_{j}^{\varepsilon / d_{2}^{3}}\right) u$

$$
\begin{gathered}
\sum_{j}\left\|U_{j}\right\|_{H_{a}^{2 m}\left(K_{j}^{\left.\varepsilon / d_{2}^{3}\right)}\right.} \leqslant c \sum_{j}\left\{\left\|f_{j}\right\|_{H_{a}^{0}\left(K_{j}^{\varepsilon}\right)}+\sum_{\sigma, \mu}\left\|f_{j \sigma \mu}\right\|_{H_{a}^{2 m-m_{j \sigma \mu}-1 / 2}\left(\gamma_{j \sigma}^{\varepsilon}\right)}\right. \\
\left.+\left\|U_{j}\right\|_{H_{a-2 m}^{0}\left(K_{j}^{\varepsilon}\right)}\right\}
\end{gathered}
$$

əде $c>0$ не зависит от $U$.

ДокАЗАТЕЛЬСтво. Обозначим $K_{j q}^{s}=K_{j} \cap\left\{\varepsilon d_{2}^{-3} d_{1}^{4-q} 2^{-s-1}<|y|<\varepsilon d_{2}^{-3} d_{2}^{4-q} 2^{-s}\right\}$, $s=0,1,2, \ldots$ Очевидно,

$$
\bigcup_{s=0}^{\infty} K_{j 1}^{s}=K_{j}^{\varepsilon}, \quad \bigcup_{s=0}^{\infty} K_{j 4}^{s}=K_{j}^{\varepsilon / d_{2}^{3}}
$$

Положим $U_{j}^{s}\left(y^{\prime}\right)=U_{j}\left(2^{-s} y^{\prime}\right)$ и сделаем замену переменных $y=2^{-s} y^{\prime}$ в уравнении

$$
\mathbf{P}_{j}\left(y, D_{y}\right) U_{j} \equiv \sum_{|\alpha| \leqslant 2 m} p_{j \alpha}(y) D_{y}^{\alpha} U_{j}(y)=f_{j}(y), \quad y \in K_{j 1}^{s}
$$

и в нелокальных условиях

$$
\left.\sum_{k, s} \sum_{|\alpha| \leqslant m_{j \sigma \mu}} b_{j \sigma \mu k s \alpha}(x) D_{x}^{\alpha} U_{j}(x)\right|_{x=\mathscr{G}_{j \sigma k s} y}=f_{j \sigma \mu}(y), \quad y \in \gamma_{j \sigma} \cap \overline{K_{j 1}^{s}}
$$

умножая обе части первого из получившихся равенств на $2^{-s \cdot 2 m}$, а второго - на $2^{-s \cdot m_{j \sigma \mu}, \text { имеем }}$

$$
\begin{gathered}
\sum_{|\alpha| \leqslant 2 m} p_{j \alpha}^{s}\left(y^{\prime}\right) 2^{s(|\alpha|-2 m)} D_{y^{\prime}}^{\alpha} U_{j}^{s}\left(y^{\prime}\right)=2^{-s \cdot 2 m} f_{j}^{s}\left(y^{\prime}\right), \quad y^{\prime} \in K_{j 1}^{0} \\
\left.\sum_{k, s} \sum_{|\alpha| \leqslant m_{j \sigma \mu}} b_{j \sigma \mu k s \alpha}^{s}\left(x^{\prime}\right) 2^{s\left(|\alpha|-m_{j \sigma \mu}\right)} D_{x^{\prime}}^{\alpha} U_{j}^{s}\left(x^{\prime}\right)\right|_{x^{\prime}=\mathscr{G}_{j \sigma k s} y^{\prime}}=2^{-s \cdot m_{j \sigma \mu}} f_{j \sigma \mu}^{s}\left(y^{\prime}\right) \\
y^{\prime} \in \gamma_{j \sigma} \cap \overline{K_{j 1}^{0}}
\end{gathered}
$$

где $p_{j \alpha}^{s}\left(y^{\prime}\right)=p_{j \alpha}\left(2^{-s} y^{\prime}\right), b_{j \sigma \mu k s \alpha}^{s}\left(x^{\prime}\right)=b_{j \sigma \mu k s \alpha}\left(2^{-s} x^{\prime}\right), f_{j}^{s}\left(y^{\prime}\right)=f_{j}\left(2^{-s} y^{\prime}\right)$ и $f_{j \sigma \mu}^{s}\left(y^{\prime}\right)$ $=f_{j \sigma \mu}\left(2^{-s} y^{\prime}\right)$. 
Применяя к задаче (2.19), (2.20) лемму 2.2, получим

$$
\begin{aligned}
\sum_{j}\left\|U_{j}^{s}\right\|_{W^{2 m}\left(K_{j 4}^{0}\right)} \leqslant & k_{1} \sum_{j}\left\{\left\|2^{-s \cdot 2 m} f_{j}^{s}\right\|_{L_{2}\left(K_{j 1}^{0}\right)}\right. \\
& \left.+\sum_{\sigma, \mu}\left\|2^{-s \cdot m_{j \sigma \mu}} f_{j \sigma \mu}^{s}\right\|_{W^{2 m-m_{j \sigma \mu}-1 / 2}\left(\gamma_{j \sigma} \cap \overline{K_{j 1}^{0}}\right)}+\left\|U_{j}^{s}\right\|_{L_{2}\left(K_{j 1}^{0}\right)}\right\}
\end{aligned}
$$

где константа $k_{1}$ в силу замечания 2.2 не зависит от $s$.

Обозначим через $\Phi_{j \sigma \mu} \in H_{a}^{2 m-m_{j \sigma \mu}}\left(K_{j}\right)$ функцию, удовлетворяющую следующим условиям: $\left.\Phi_{j \sigma \mu}\right|_{\gamma_{j \sigma}^{\varepsilon}}=f_{j \sigma \mu}$ и

$$
\left\|\Phi_{j \sigma \mu}\right\|_{H_{a}^{2 m-m_{j \sigma \mu}}\left(K_{j}^{\varepsilon}\right)} \leqslant 2\left\|f_{j \sigma \mu}\right\|_{H_{a}^{2 m-m_{j \sigma \mu}-1 / 2}\left(\gamma_{j \sigma}^{\varepsilon}\right)}
$$

(существование такой функции вытекает из (2.10)). Тогда $\left.\Phi_{j \sigma \mu}^{s}\right|_{\gamma_{j \sigma} \cap \overline{K_{j 1}^{0}}}=f_{j \sigma \mu}^{s}$, где $\Phi_{j \sigma \mu}^{s}\left(y^{\prime}\right)=\Phi_{j \sigma \mu}\left(2^{-s} y^{\prime}\right)$. Следовательно, из (2.21) и (1.3) получаем

$$
\begin{aligned}
\sum_{j}\left\|U_{j}^{s}\right\|_{W^{2 m}\left(K_{j 4}^{0}\right)} \leqslant & k_{1} \sum_{j}\left\{\left\|2^{-s \cdot 2 m} f_{j}^{s}\right\|_{L_{2}\left(K_{j 1}^{0}\right)}\right. \\
& \left.+\sum_{\sigma, \mu}\left\|2^{-s \cdot m_{j \sigma \mu}} \Phi_{j \sigma \mu}^{s}\right\|_{W^{2 m-m_{j \sigma \mu}\left(K_{j 1}^{0}\right)}}+\left\|U_{j}^{s}\right\|_{L_{2}\left(K_{j 1}^{0}\right)}\right\} .
\end{aligned}
$$

Делая в неравенстве (2.23) обратную замену переменньх $y^{\prime}=2^{s} y$, получим

$$
\begin{aligned}
& \sum_{j} \sum_{|\alpha| \leqslant 2 m}\left\|2^{-s|\alpha|} D_{y}^{\alpha} U_{j}\right\|_{L_{2}\left(K_{j 4}^{s}\right)} \leqslant k_{1} \sum_{j}\left\{\left\|2^{-s \cdot 2 m} f_{j}\right\|_{L_{2}\left(K_{j 1}^{s}\right)}\right. \\
& \left.\quad+\sum_{\sigma, \mu|\alpha| \leqslant 2 m-m_{j \sigma \mu}}\left\|2^{-s\left(|\alpha|+m_{j \sigma \mu}\right)} \Phi_{j \sigma \mu}\right\|_{L_{2}\left(K_{j 1}^{s}\right)}+\left\|U_{j}\right\|_{L_{2}\left(K_{j 1}^{s}\right)}\right\} .
\end{aligned}
$$

Умножая неравенство $(2.24)$ на $2^{-s(a-2 m)}$, суммируя по $s$ и учитывая $(2.22)$ и $(2.18)$, выводим (2.17).

Лемма 2.3 совместно с леммой 2.1 дает $u \in H_{a}^{2 m}(G), a>2 m-1$, где $u$ - произвольное обобщенное решение задачи $(1.8),(1.9)$ с правой частью $f_{0} \in L_{2}(G)$.

Из [16, лемма 2.1] и [17, теорема 3.2$]$ следует, что при почти всех $a>2 m-1$ множество решений из $H_{a}^{2 m}(G)$ задачи $(1.8),(1.9)$ с правой частью $f_{0}=0$ образует конечномерное пространство. Таким образом, доказан следуюший результат.

Лемма 2.4. Пусть выполнены условия 1.1 и 1.2. Тогда ядро оператора $\mathbf{P}$ конечномерно. 
2.2. Замкнутость оператора и его образа. Конечномерность коядра. Для доказательства фредгольмовости оператора $\mathbf{P}$ нам потребуется рассмотреть задачу $(1.8),(1.9)$ в пространствах с весом $a$ таким, что $0<a \leqslant m$. Трудность здесь заключается в том, что из принадлежности $u \in H_{a}^{2 m}(G)$ теперь, вообе говоря, не следует, что $\mathbf{B}_{i \mu}^{2} u$ принадлежит $H_{a}^{2 m-m_{i \mu}-1 / 2}\left(\Gamma_{i}\right) ;$ поэтому и сумма $\mathbf{B}_{i \mu} u=\mathbf{B}_{i \mu}^{0} u+\mathbf{B}_{i \mu}^{1} u+\mathbf{B}_{i \mu}^{2} u$ может не принадлежать $H_{a}^{2 m-m_{i \mu}-1 / 2}\left(\Gamma_{i}\right)$. Можно лишь гарантировать, что $\mathbf{B}_{i \mu} u \in H_{a^{\prime}}^{2 m-m_{i \mu}-1 / 2}\left(\Gamma_{i}\right), a^{\prime}>2 m-1$ (это следует из принадлежности $\mathbf{B}_{i \mu} u \in W^{2 m-m_{i \mu}-1 / 2}\left(\Gamma_{i}\right)$ и [26, лемма 5.2]). Однако в [23, §6] доказано, что

$$
\left\{\mathbf{P}\left(y, D_{y}\right) u, \mathbf{B}_{i \mu} u\right\} \in \mathscr{H}_{a}^{0}(G, \Gamma) \dot{+} \mathscr{R}_{a}^{0}(G, \Gamma) \quad \text { при всех } u \in H_{a}^{2 m}(G), \quad a>0,
$$

где $\mathscr{H}_{a}^{0}(G, \Gamma)=H_{a}^{0}(G) \times \prod_{i, \mu} H_{a}^{2 m-m_{i \mu}-1 / 2}\left(\Gamma_{i}\right)$, а $\mathscr{R}_{a}^{0}(G, \Gamma)$ - некоторое конечномерное пространство, вложенноев $\{0\} \times \prod_{i, \mu} H_{a^{\prime}}^{2 m-m_{i \mu}-1 / 2}\left(\Gamma_{i}\right)$ при любом $a^{\prime}>2 m-1$, т.е. пространство $\mathscr{R}_{a}^{0}(G, \Gamma)$ содержит лишш функции вида $\left\{0, f_{i \mu}\right\}, f_{i \mu} \in H_{a^{\prime}}^{2 m-m_{i \mu}-1 / 2}\left(\Gamma_{i}\right)$, $f_{i \mu} \notin H_{a}^{2 m-m_{i \mu}-1 / 2}\left(\Gamma_{i}\right)$. Зафиксируем некоторое число $a^{\prime}>2 m-1$. Тогда любая функция $\left\{f_{0}, f_{i \mu}\right\} \in \mathscr{H}_{a}^{0}(G, \Gamma) \dot{+} \mathscr{R}_{a}^{0}(G, \Gamma)$ представима единственным образом в виде $\left\{f_{0}, f_{i \mu}\right\}=\left\{f_{0}, f_{i \mu}^{1}\right\}+\left\{0, f_{i \mu}^{2}\right\}$, где $\left\{f_{0}, f_{i \mu}^{1}\right\} \in \mathscr{H}_{a}^{0}(G, \Gamma),\left\{0, f_{i \mu}^{2}\right\} \in \mathscr{R}_{a}^{0}(G, \Gamma)$, и ее норма равна

$$
\left\|\left\{f_{0}, f_{i \mu}\right\}\right\|_{\mathscr{H}_{a}^{0}(G, \Gamma) \dot{+} \mathscr{R}_{a}^{0}(G, \Gamma)}=\left(\left\|\left\{f_{0}, f_{i \mu}^{1}\right\}\right\|_{\mathscr{H}_{a}^{0}(G, \Gamma)}^{2}+\sum_{i, \mu}\left\|f_{i \mu}^{2}\right\|_{H_{a^{\prime}}^{2 m-m_{i \mu}-1 / 2}\left(\Gamma_{i}\right)}^{2}\right)^{1 / 2} .
$$

Более того, по теореме 6.1 из [23] оператор

$$
\mathbf{L}_{a}=\left\{\mathbf{P}\left(y, D_{y}\right), \mathbf{B}_{i \mu}\right\}: H_{a}^{2 m}(G) \rightarrow \mathscr{H}_{a}^{0}(G, \Gamma) \dot{+} \mathscr{R}_{a}^{0}(G, \Gamma), \quad a>0,
$$

фредгольмов при почти всех $a>0$. Другими словами, если $u \in H_{a}^{2 m}(G)$, то $\mathbf{L}_{a} u$ "принадлежит" пространству $\mathscr{H}_{a}^{0}(G, \Gamma)$ с точностью до функции вида $\left\{0, f_{i \mu}\right\}$ из конечномерного пространства $\mathscr{R}_{a}^{0}(G, \Gamma)$.

Используя оператор $\mathbf{L}_{a}$, докажем следующий результат.

Лемма 2.5. Пусть выполнены условия 1.1 и 1.2. Тогда оператор $\mathbf{P}$ замкнут, образ $\mathscr{R}(\mathbf{P})$ замкнут $и \operatorname{codim} \mathscr{R}(\mathbf{P})<\infty$.

ДокАЗАТЕЛЬСтво. 1) Рассмотрим при $0<a \leqslant m$ вспомогательный неограниченный оператор $\mathbf{P}_{a}: \operatorname{Dom}\left(\mathbf{P}_{a}\right) \subset L_{2}(G) \rightarrow L_{2}(G)$, действуюший по формуле

$$
\mathbf{P}_{a} u=\mathbf{P}\left(y, D_{y}\right) u, \quad u \in \operatorname{Dom}\left(\mathbf{P}_{a}\right)=\left\{u \in H_{a}^{2 m}(G): \mathbf{B}_{i \mu} u=0, \mathbf{P}\left(y, D_{y}\right) u \in L_{2}(G)\right\} .
$$

Зафиксируем такое $a, 0<a \leqslant m$, чтобы оператор $\mathbf{L}_{a}$ был фредгольмов. Покажем, что тогда и оператор $\mathbf{P}_{a}$ будет фредгольмов.

Из фредгольмовости $\mathbf{L}_{a}$, компактности вложения $H_{a}^{2 m}(G) \subset H_{a}^{0}(G)$ (см. [15, лемма 3.5]) и теоремы 7.1 из [27] следует, что для всех $u \in H_{a}^{2 m}(G)$

$$
\|u\|_{H_{a}^{2 m}(G)} \leqslant k_{1}\left(\left\|\mathbf{L}_{a} u\right\|_{\mathscr{H}_{a}^{0}(G, \Gamma) \dot{+} \mathscr{R}_{a}^{0}(G, \Gamma)}+\|u\|_{H_{a}^{0}(G)}\right) .
$$


Пусть теперь $u \in \operatorname{Dom}\left(\mathbf{P}_{a}\right) ;$ тогда $\mathbf{L}_{a} u=\left\{\mathbf{P}\left(y, D_{y}\right) u, 0\right\}, \mathbf{P}\left(y, D_{y}\right) u \in L_{2}(G) \subset H_{a}^{0}(G)$ и, следовательно,

$$
\left\|\mathbf{L}_{a} u\right\|_{\mathscr{H}_{a}^{0}(G, \Gamma) \dot{+} \mathscr{R}_{a}^{0}(G, \Gamma)}=\left\|\mathbf{P}\left(y, D_{y}\right) u\right\|_{H_{a}^{0}(G)} .
$$

Отсюда и из $(2.25)$, учитьвая непрерьвность вложения $L_{2}(G) \subset H_{a}^{0}(G)$ при $a>0$, получим

$$
\|u\|_{H_{a}^{2 m}(G)} \leqslant k_{2}\left(\left\|\mathbf{P}\left(y, D_{y}\right) u\right\|_{H_{a}^{0}(G)}+\|u\|_{H_{a}^{0}(G)}\right) \leqslant k_{3}\left(\left\|\mathbf{P}\left(y, D_{y}\right) u\right\|_{L_{2}(G)}+\|u\|_{L_{2}(G)}\right),
$$

где $u \in \operatorname{Dom}\left(\mathbf{P}_{a}\right)$. Из неравенства (2.26) вытекает, что оператор $\mathbf{P}_{a}$ замкнут. Следовательно, снова используя (2.26) и теорему 7.1 из [27], получим что $\operatorname{dim} \operatorname{ker} \mathbf{P}_{a}<\infty$ (ясно, что $\left.\operatorname{ker} \mathbf{P}_{a}=\operatorname{ker} \mathbf{L}_{a}\right)$ и $\mathscr{R}\left(\mathbf{P}_{a}\right)$ замкнут.

Рассмотрим произвольную функцию $f_{0} \in L_{2}(G)$. Очевидно, $f_{0} \in H_{a}^{0}(G)$. В силу [23, следствие 6.1] существуют функционалы $F_{1}, \ldots, F_{q_{0}}$ из сопряженного пространства $\mathscr{H}_{a}^{0}(G, \Gamma)^{*}$ такие, что если $\left\langle\left\{f_{0}, 0\right\}, F_{q}\right\rangle=0, q=1, \ldots, q_{0}$, то задача (1.8), (1.9) имеет решение $u \in H_{a}^{2 m}(G)$. Так как

$$
\left|\left\langle\left\{f_{0}, 0\right\}, F_{q}\right\rangle\right| \leqslant k_{4}\left\|f_{0}\right\|_{H_{a}^{0}(G)} \leqslant k_{5}\left\|f_{0}\right\|_{L_{2}(G)},
$$

по теореме Рисса об общем виде линейного непрерьвного функционала в гильбертовом пространстве существуют функции $f_{1}, \ldots, f_{q_{0}} \in L_{2}(G)$ такие, что $\left\langle\left\{f_{0}, 0\right\}, F_{q}\right\rangle=$ $\left(f_{0}, f_{q}\right)_{L_{2}(G)}, q=1, \ldots, q_{0}$. Следовательно, $\operatorname{codim} \mathscr{R}\left(\mathbf{P}_{a}\right) \leqslant q_{0}$. Итак, оператор $\mathbf{P}_{a}$ фредгольмов.

2) Поскольку $H_{a}^{2 m}(G) \subset H_{a-m}^{m}(G) \subset W^{m}(G)$ при $a \leqslant m$, имеет место соотношение

$$
\mathbf{P}_{a} \subset \mathbf{P} \text {. }
$$

Из (2.27), в частности, следует, что образ $\mathscr{R}(\mathbf{P})$ замкнут и $\operatorname{codim} \mathscr{R}(\mathbf{P}) \leqslant \operatorname{codim} \mathscr{R}\left(\mathbf{P}_{a}\right)$ $\leqslant q_{0}$.

Осталось доказать, что $\mathbf{P}$ замкнут $^{2}$. Обозначим через $h_{1}, \ldots, h_{k}$ базис в пространстве

$$
\mathscr{R}\left(\mathbf{P}_{a}\right)^{\perp}=\mathscr{R}(\mathbf{P}) \ominus \mathscr{R}\left(\mathbf{P}_{a}\right) .
$$

Тогда существуют функции $v_{1}, \ldots, v_{k} \in \operatorname{Dom}(\mathbf{P})$ такие, что $\mathbf{P} v_{j}=h_{j}, j=1, \ldots, k$. Так как $h_{j} \notin \mathscr{R}\left(\mathbf{P}_{a}\right)$, то $v_{j} \notin \operatorname{Dom}\left(\mathbf{P}_{a}\right)$. Ясно также, что функции $v_{1}, \ldots, v_{k}$ линейно независимы, так как линейно независимы функции $h_{1}, \ldots, h_{k}$.

Рассмотрим конечномерное пространство

$$
\mathscr{N}=\operatorname{Span}\left(v_{1}, \ldots, v_{k}, \operatorname{ker} \mathbf{P}\right) \ominus \operatorname{ker} \mathbf{P}_{a} .
$$

\footnotetext{
${ }^{2}$ Отметим, что из замкнутости образа некоторого оператора $\mathbf{P}$ в гильбертовом пространстве и конечномерности его ядра и коядра, вообще говоря, не следует замкнутость самого оператора $\mathbf{P}$; это можно показать, используя рассуждения, близкие к приведенным, например, в [28, гл. 2, п. 18]. Однако если дополнительно предположить, что оператор $\mathbf{P}$ является расширением фредгольмова оператора, то, как мы увидим, $\mathbf{P}$ будет замкнут.
} 
Нетрудно видеть, что $\mathscr{N} \cap \operatorname{Dom} \mathbf{P}_{a}=\{0\}$. Действительно, если $u \in \mathscr{N} \cap \operatorname{Dom} \mathbf{P}_{a}$, то

$$
u=\sum_{i=1}^{k} \alpha_{i} v_{i}+v
$$

где $\alpha_{i}-$ некоторые константы, $v \in \operatorname{ker} \mathbf{P}$. Тогда, учитьвая $(2.27)$, имеем

$$
\sum_{i=1}^{k} \alpha_{i} h_{i}=\mathbf{P} u=\mathbf{P}_{a} u \in \mathscr{R}\left(\mathbf{P}_{a}\right) .
$$

Следовательно, $\alpha_{i}=0, i=1, \ldots, k$, и, значит, $u=v$. Снова используя (2.27), видим, что $u=v \in \operatorname{ker} \mathbf{P}_{a}$. Отсюда, по определению пространства $\mathscr{N}$, получаем $u=0$.

Обозначим через $\operatorname{Gr} \mathbf{P}\left(\operatorname{Gr} \mathbf{P}_{a}\right)$ график оператора $\mathbf{P}\left(\mathbf{P}_{a}\right)$. Как известно, оператор $\mathbf{P}$ $\left(\mathbf{P}_{a}\right)$ замкнут тогда и только тогда, когда замкнут его график $\operatorname{Gr} \mathbf{P}\left(\operatorname{Gr} \mathbf{P}_{a}\right)$ в $L_{2}(G) \times L_{2}(G)$.

Так как $\operatorname{Gr} \mathbf{P}_{a}$ замкнут (как график замкнутого оператора) и $\operatorname{Gr} \mathbf{P}_{a} \subset \mathrm{Gr} \mathbf{P}$, а пространства $\mathscr{N}$ и $\mathscr{R}\left(\mathbf{P}_{a}\right)^{\perp}$ конечномерны, то для доказательства замкнутости оператора $\mathbf{P}$ достаточно показать, что

$$
\operatorname{Gr} \mathbf{P} \subset \operatorname{Gr} \mathbf{P}_{a} \dot{+}\left(\mathscr{N} \times \mathscr{R}\left(\mathbf{P}_{a}\right)^{\perp}\right) .
$$

Очевидно, сумма в (2.28) действительно является прямой суммой: если

$$
(u, f) \in \operatorname{Gr} \mathbf{P}_{a} \cap\left(\mathscr{N} \times \mathscr{R}\left(\mathbf{P}_{a}\right)^{\perp}\right),
$$

то $u \in \operatorname{Dom} \mathbf{P}_{a} \cap \mathcal{N}=\{0\}$ и, следовательно, $(u, f)=\left(u, \mathbf{P}_{a} u\right)=(0,0)$.

Далее, пусть $(u, f) \in \operatorname{Gr} \mathbf{P}$, т.е. $u \in \operatorname{Dom} \mathbf{P}$ и $f=\mathbf{P} u$. Представим функцию $f$ в виде суммы

$$
f=f_{1}+f_{2},
$$

где $f_{1} \in \mathscr{R}\left(\mathbf{P}_{a}\right), f_{2} \in \mathscr{R}\left(\mathbf{P}_{a}\right)^{\perp}$. Выберем элемент $u_{1} \in \operatorname{Dom}\left(\mathbf{P}_{a}\right)$ такой, что $\mathbf{P}_{a} u_{1}=f_{1}$. Тогда $u_{2}=u-u_{1} \in \operatorname{Dom}(\mathbf{P})$ и $\mathbf{P} u_{2}=f_{2}$. Без ограничения обшности можем считать, что

$$
u_{2} \perp \operatorname{ker} \mathbf{P}_{a} ;
$$

если это соотношение не выполнено, то следует рассмотреть проекцию $u_{2 a}$ функции $u_{2}$ на $\operatorname{ker} \mathbf{P}_{a}$ и заменить $u_{1}$ на $u_{1}+u_{2 a}$, а $u_{2}-$ на $u_{2}-u_{2 a}$. Очевидно, $\left(u_{1}, f_{1}\right) \in \operatorname{Gr} \mathbf{P}_{a}$ и, учитывая $(2.29),\left(u_{2}, f_{2}\right) \in \mathscr{N} \times \mathscr{R}\left(\mathbf{P}_{a}\right)^{\perp}$. Таким образом, соотношение $(2.28)$ и вся лемма доказаны.

Из лемм 2.4 и 2.5 вытекает теорема 2.1.

ЗАмечАнИЕ 2.3. Используя результаты работы [29], можно показать, что теорема 2.1 справедлива и в том случае, когда преобразования $\Omega_{i s}$ нелинейны вблизи точек множества $\mathscr{K}$, а их линейные части удовлетворяют условию 1.1 .

Автор выражает благодарность профессору А. Л. Скубачевскому за внимание к работе. 


\section{СПИСОК ЦИТИРОВАННОЙ ЛИТЕРАТУРЫ}

[1] Sommerfeld A. Ein Beitrag zur hydrodinamischen Erklärung der turbulenten Flussigkeitsbewegungen // Proc. Intern. Congr. Math. (Rome, 1908). V. 3. Reale Accad. Lincei: Roma, 1909. P. 116-124.

[2] Тамаркин Я. Д. О некоторых общих задачах теории обькновенных линейных дифференциальных уравнений. Петроград, 1917.

[3] Picone M. Equazione integrale traducente il più generale problema lineare per le equazioni differenziali lineari ordinarie di qualsivoglia ordine // Acad. Nazionale Lincei. Atti dei Convegni. 1932. V. 15. P. 942-948.

[4] Carleman T. Sur la théorie des equations integrales et ses applications // Verhandlungen des Internat. Math. Kongress. V. 1. Zürich, 1932. P. 132-151.

[5] Бицадзе А. В., Самарский А. А. О некоторых простейших обобщениях линейных эллиптических краевых задач // Докл. АН СССР. 1969. Т. 185. №4. С. 739-740.

[6] Вишик М. И. Об общих краевых задачах для эллиптических дифференциальных уравнений // Tp. MMO. 1952. T. 1. C. 187-246.

[7] Browder F. Non-local elliptic boundary value problems // Amer. J. Math. 1964. V. 86. P. $735-750$.

[8] Жиитарашу Н. В., Эйдельман С. Д. О нелокальных граничных задачах для эллиптических уравнений // Матем. исслед. 1971. Т. 6. №2 20). С. 63-73.

[9] Ройтберг Я. А., Шефтель З. Г. Нелокальные задачи для эллиптических уравнений и систем // Сиб. матем. ж. 1972. Т. 13. № 1. С. 165-181.

[10] Кишкис К. Ю. Об индексе задачи Бицадзе-Самарского для гармонических функций // Дифференц. уравнения. 1988. Т. 24. №1. С. 105-110.

[11] Гущин А. К., Михайлов В. П. О разрешимости нелокальных задач для эллиптических уравнений второго порядка // Матем. сб. 1994. Т. 185. №1. С. 121-160.

[12] Гущин А. К. Условие компактности одного класса операторов и его применение к исследованию разрешимости нелокальных задач для эллиптических уравнений // Матем. сб. 2002. T. 193. № 5. C. 17-36.

[13] Скубачевский А. Л. Эллиптические задачи с нелокальньми условиями вблизи границы // Матем. сб. 1986. Т. 129(171). № 2. С. 279-302.

[14] Skubachevskii A. L. Regularity of solutions for some nonlocal elliptic problem // Russian J. Math. Phys. 2001. V. 8. P. 365-374.

[15] Кондратьев В. А. Краевые задачи для эллиптических уравнений в областях с коническими или угловыми точками // Тр. ММО. 1967. Т. 16. С. 209-292.

[16] Скубачевский А. Л. Модельные нелокальные задачи для эллиптических уравнений в двугранных углах // Дифференц. уравнения. 1990. Т. 26. №1. С. 120-131.

[17] Скубачевский А. Л. О методе срезающих функций в теории нелокальных задач // Дифференц. уравнения. 1991. Т. 27. №1. С. 128-139.

[18] Skubachevskii A. L. On the stability of index of nonlocal elliptic problems // J. Math. Anal. Appl. 1991. V. 160. № 2. P. 323-341.

[19] Skubachevskii A. L. Elliptic Functional Differential Equations and Applications. BaselBoston-Berlin: Birkhäuser, 1997.

[20] Feller W. Diffusion processes in one dimension // Trans. Amer. Math. Soc. 1954. V. 77. P. 1-30.

[21] Самарский А. А. О некоторых проблемах теории дифференциальных уравнений // Дифференц. уравнения. 1980. Т. 16. №11. С. 1925-1935.

[22] Gurevich P. L. Solvability of nonlocal elliptic problems in Sobolev spaces, I // Russian J. Math. Phys. 2003. V. 10. № 4. P. 436-466.

[23] Gurevich P. L. Solvability of nonlocal elliptic problems in Sobolev spaces, II // Russian J. Math. Phys. 2004. V. 11. № 1. P. 1-44.

[24] Скубачевский А. Л. О некоторых нелокальных эллиптических краевых задачах // Дифференц. уравнения. 1982. Т. 18. №9. С. 1590-1599. 
[25] Лионс Ж.-Л., Мадженес Э. Неоднородные граничные задачи и их приложения. М.: Мир, 1971.

[26] Ковалева О. А., Скубачевский А. Л. Разрешимость нелокальных эллиптических задач в пространствах с весом // Матем. заметки. 2000. Т. 67. №6. С. 882-898.

[27] Крейн С. Г. Линейные уравнения в банаховом пространстве. М.: Наука, 1971.

[28] Ахиезер Н. И., Глазман И. М. Теория линейных операторов в гильбертовом пространстве. М.: Наука, 1966.

[29] Гуревич П. Л. Нелокальные эллиптические задачи с нелинейными преобразованиями переменных вблизи точек сопряжения // Изв. РАН. Сер. матем. 2003. Т. 67. №6. С. 81-120.

Московский авиационный институт

Поступило

E-mail: gurevichp@yandex.ru 